\title{
EXPRESSION OF COX-2 IN HUMAN BREAST CANCER CELLS AS A CRITICAL DETERMINANT OF EPITHELIAL-MESENCHYMAL TRANSITION AND INVASIVENESS
}

\section{This is the author's manuscript}

Original Citation:

Availability:

This version is available http://hdl.handle.net/2318/139953

since 2016-07-26T10:41:02Z

Published version:

DOI:10.1517/14728222.2014.860447

Terms of use:

Open Access

Anyone can freely access the full text of works made available as "Open Access". Works made available under a Creative Commons license can be used according to the terms and conditions of said license. Use of all other works requires consent of the right holder (author or publisher) if not exempted from copyright protection by the applicable law. 


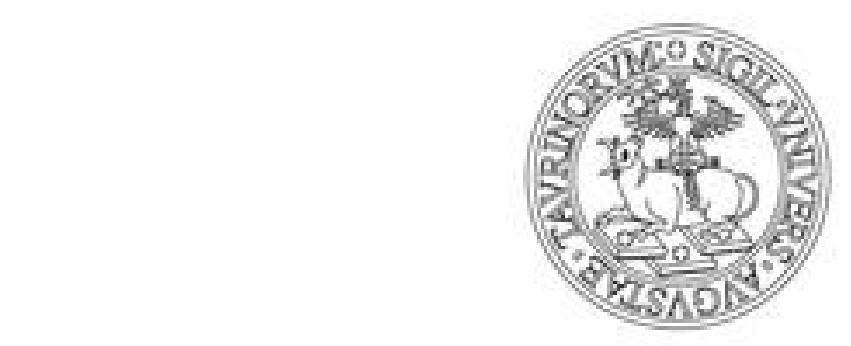

\section{UNIVERSITÀ DEGLI STUDI DI TORINO}

This is an author version of the contribution published on:

C. BOCCA, M. IEVOLELLA, R. AUTELLI, M. MOTTA, L. MOSSO, B. TORCHIO, F. BOZZO, S. CANNITO, C. PATERNOSTRO, S. COLOMBATTO, M. PAROLA, A. MIGLIETTA EXPRESSION OF COX-2 IN HUMAN BREAST CANCER CELLS AS A CRITICAL DETERMINANT OF EPITHELIAL-MESENCHYMAL TRANSITION AND INVASIVENESS EXPERT OPINION ON THERAPEUTIC TARGETS (2014) 18 DOI: $10.1517 / 14728222.2014 .860447$

The definitive version is available at:

http://informahealthcare.com/doi/abs/10.1517/14728222.2014.860447 
EXPRESSION OF COX-2 IN HUMAN BREAST CANCER CELLS AS A CRITICAL

DETERMINANT OF EPITHELIAL-MESENCHYMAL TRANSITION AND INVASIVENESS

BOCCA CLAUDIA*, IEVOLELLA MONICA, AUTELLI RICCARDO, MOTTA MANUELA ${ }^{1}$, MOSSO LUCIANO ${ }^{1}$, TORCHIO BRUNO ${ }^{1}$, BOZZO FRANCESCA, CANNITO STEFANIA, PATERNOSTRO CLAUDIA, COLOMBATTO SEBASTIANO ${ }^{2}$, PAROLA MAURIZIO, MIGLIETTA ANTONELLA

University of Torino, Department of Clinical and Biological Sciences - C.so Raffaello, 30 - 10125

Torino, Italy; ${ }^{1}$ A.O. Ordine Mauriziano, Pathology Unit - Largo Turati 62 - 10128 Torino, Italy; ${ }^{2}$ University of Torino, Department of Oncology - Via Michelangelo, 27 - 10125 Torino, Italy

*CORRESPONDING AUTHOR:

Department of Clinical and Biological Sciences - University of Torino

C.so Raffaello, 30 - 10125 Torino, Italy

Tel: +39 0116707756

Fax: +39 0116707753

e-mail: claudia.bocca@unito.it 


\section{ABSTRACT}

Introduction: Cyclooxygenase-2 (COX-2) is overexpressed in several malignancies and is implicated in breast cancer progression. Objectives: We investigated whether changes in COX-2 expression may affect epithelial-to-mesenchymal transition (EMT) and then invasive potential of human breast cancer cells, in relationship with hypoxia. COX-2-null MCF-7 human breast cancer cells, MCF-7 cells transiently expressing COX-2, and COX-2-expressing MDA-MB-231 cells were employed. Results: COX-2 overexpression resulted in down-regulation of E-cadherin and Bcatenin, up-regulation of vimentin, N-cadherin and SNAI1, suggesting EMT occurrence. COX-2overexpressing MCF-7 cells were also characterized by increased invasiveness and release of matrix-metalloproteinase-9. The above-mentioned characteristics, homologous to those detected in highly invasive MDA-MB-231 cells, were reverted by treatment of COX-2-overexpressing MCF-7 cells with celecoxib, a COX-2 specific inhibitor, partly through the inhibition of COX-2-related intracellular generation of reactive oxygen species. Hypoxia further exacerbated COX-2 expression, EMT changes and invasive ability in both COX-2-overexpressing MCF-7 cells and MDA-MB-231 cells. Finally, immunohistochemistry performed on samples from normal and neoplastic human breast tissues revealed that COX-2-positive malignant cells were also positive for EMT-related antigens, HIF-2 $\alpha$ and the oxidative stress marker heme-oxygenase. Conclusions: These findings support the existence of a direct link between COX-2 overexpression, EMT and invasiveness in human breast cancer cells, emphasizing the role of hypoxic microenvironment.

KEY WORDS: COX-2; EMT; invasiveness; hypoxia; MCF-7 cells; MDA-MB-231 cells; ROS 


\section{INTRODUCTION}

Breast cancer is a leading cause of death in females in the western world [1]. In the last decade several studies have outlined a link between the pathogenesis of breast cancer and the expression of cyclooxygenase-2 (COX-2) [2-5]. Deregulation of COX-2 expression leads to an increased abundance of prostaglandin $\mathrm{E}_{2}\left(\mathrm{PGE}_{2}\right)$, which can potentially affect most of key processes in cancer development, including proliferation, resistance to apoptosis, angiogenesis, immune suppression and invasion $[6,7]$.

COX-2 expression in invasive breast cancer as well as its crucial role in the ability of carcinoma in situ to progress towards invasive breast cancer has been widely documented and likely depends on COX-2 role in the destruction of the basal membrane and in the formation of new blood vessels allowing tumour growth [8]. Thus, COX-2 expression has been associated with negative prognosis and disease progression [9].

According to literature, also the tumour microenvironment can play a crucial role in cancer progression [10], with fluctuation of hypoxia and nutrient deprivation being prominent features which can lead to cellular adaptation. Cell adaptation can favour survival of cancer cells and can give rise to heterogeneity and selection of resistant clones, then resulting in a more malignant phenotype [11]. Along these lines, hypoxia is indeed a common feature of solid malignant tumours and is predominantly caused by abnormal vascularization of the rapidly growing tumour mass, with areas of low oxygen pressure being heterogeneously distributed [12,13].

The key regulators of cellular responses to hypoxia are hypoxia-inducible factors (HIFs) which are known to activate and/or regulate target genes involved in a number of different cellular processes, including proliferation, angiogenesis and epithelial-to-mesenchymal transition (EMT), all linked to malignant progression $[11,14,15]$.

EMT has emerged in recent years as a fundamental biological process, leading epithelial cells to lose their polarization and specialized junctional structures, to undergo cytoskeleton reorganization, and to acquire morphological and functional features of mesenchymal-like cells [16-19]. 
One of the most convincing evidence for EMT in the clinical scenario involves the loss of selected epithelial features in cancer cells, typically involving the adherent junction proteins E-cadherin, $\alpha$ and $\beta$-catenin, and cytokeratins, resulting in destabilisation of cell-cell contacts and detachment of cells from surrounding ones [20]. Concomitantly, a number of mesenchymal markers are increased in their expression, including $\mathrm{N}$-cadherin, vimentin, fibronectin, matrix metalloproteinases as well as the transcriptional regulator of the mesenchymal phenotype SNAI1 [21,22] .

Along these lines, it has been proposed that COX-2 may be induced by the hypoxic microenvironment in colorectal and lung cancer cells via HIF-1-dependent mechanisms [23,24], an event that may enhance cell motility and invasion. Although the mechanisms underlying this scenario are still not entirely clear, hypoxia-related COX-2 up-regulation is associated with an increase in $\mathrm{PGE}_{2}$ levels [24], suggesting a role for $\mathrm{PGE}_{2}$ in the promotion of cell survival under hypoxic conditions, a property that tumour cells acquire in order to propagate and progress [15].

The present study sought to investigate the influence of the COX-2/PGE 2 pathway on the behaviour of breast cancer cells, focusing the attention on the hypothesis that COX-2 up-regulation may be linked to EMT and, therefore, critical for motility, invasion, and metastasis. In order to explore this hypothesis we created a model of human breast cancer cells overexpressing COX-2, by means of transient transfection of human COX-2 cDNA into the COX-2 null [25], poorly invasive and estrogen-dependent MCF-7 human breast cancer cell line. Furthermore, COX-2 expression in human breast cancer malignancy was preliminarly examined in a panel of clinical specimens from human breast cancers.

\section{MATERIALS AND METHODS}

\subsection{Materials and reagents}

Mouse monoclonal antibody for $\beta$-actin and anti-goat Cy3-conjugated secondary antibody were purchased from Sigma Chemical Co (MO, USA). Rabbit polyclonal antibodies for p-Akt, pERK1/2, N-cadherin, E-cadherin, SNAI1, HIF-1 $\alpha, \beta$-catenin, vimentin and HRP-conjugated 
secondary antibodies were purchased from Santa Cruz Biotechnology, Inc. (CA, USA). Rabbit polyclonal antibodies for $\mathrm{HO}-1$ and $\mathrm{COX}-2$ were from Stressgen Biotechnologies (British Columbia, Canada) and Cayman Chemical (Ann Arbor, MI, USA), respectively. Rabbit polyclonal antibody for HIF-2 $\alpha$ was from Novus Biologicals (Cambridge, UK). Anti-rabbit Cy3-conjugated secondary antibody was from GE Healthcare Europe GmbH (Milano, Italy). Boyden chambers were from Neuro Probe Inc. (MD, USA). The X-treme GENE HP DNA transfection reagent was from Roche Diagnostics (Mannheim, Germany). Celecoxib was obtained from Sequoia Research Products Ltd (Pangbourne, UK), solubilized in dimethyl sulfoxide (DMSO) and used at a final concentration of DMSO that never exceeded $0.1 \%$. The enhanced chemiluminescence reagent and nitrocellulose membrane (Hybond-C extra) were from GE Healthcare Europe GmbH (Milano, Italy). All other reagents were from Sigma Chemical Co (MO, USA) .

\subsection{Cell lines and culture conditions}

MCF-7 and MDA-MB-231 human breast cancer cells, from American Type Cell Culture (ATCC, Manassas, VA, USA), were grown and maintained in DMEM medium supplemented with 10\% foetal bovine serum, $100 \mathrm{U} / \mathrm{ml}$ penicillin, $100 \mu \mathrm{g} / \mathrm{ml}$ streptomycin and $25 \mu \mathrm{g} / \mathrm{ml}$ amphotericin B. Cells were cultured at $37{ }^{\circ} \mathrm{C}$ in a humidified incubator with $5 \% \mathrm{CO}_{2}$ and $95 \%$ air, and regularly examined using inverted microscope.

In most of the experiments cells were seeded at $3 \times 10^{4}$ cells $/ \mathrm{cm}^{2}$ density and cultured in normoxic conditions for $24 \mathrm{~h}$ to allow them to adhere. In experiments designed to evaluate the role of hypoxia, MCF-7 cells, COX-2-overexpressing MCF-7 cells and MDA-MB-231 cells were first seeded in normoxic conditions to obtain the desired subconfluence level $(65-70 \%)$ and then incubated under strictly controlled hypoxic conditions $\left(3 \% \mathrm{O}_{2}\right)$ for $2 \mathrm{~h}, 4 \mathrm{~h}, 8 \mathrm{~h}$ and $24 \mathrm{~h}[21]$.

\subsection{Transient expression of $\mathrm{COX}-2$}

For transfections, the COX-2 cDNA cloned in the pCMV-Sport6 (Source Bioscience, Nottingham, UK) was used. MCF-7 cells were seeded onto 6-well plates; after $24 \mathrm{~h}$, the cells were transfected with $2 \mu \mathrm{g}$ DNA/well of either the plasmid encoding the wild-type COX-2, or with the empty vector, 
using X-treme GENE HP DNA transfection reagent according to manufacturer's recommendations. Cells were allowed to grow 24 or $48 \mathrm{~h}$ after transfection before harvesting for the assays.

\subsection{Detection of prostaglandin $E_{2}$}

Cells plated at $6.5 \times 10^{5}$ cells in F25 flasks were grown in $10 \%$ heat-inactivated FBS for $24 \mathrm{~h}$, washed with PBS and serum starved for $24 \mathrm{~h}$. Cells were exposed for 30 min to $10 \mu \mathrm{M}$ sodium arachidonate (Sigma Chemical Co, MO, USA); then, the medium was collected from culture plates to evaluate $\mathrm{PGE}_{2}$ production using an Enzyme Immunoassay (EIA) according to manufacturer's instructions (Cayman Chemical, Ann Arbor, MI).

\subsection{Viability assay}

Cells were seeded in 12-well culture plates and treated as required. Cells were incubated with trypan blue solution $(0.5 \%$ in $\mathrm{NaCl})$ for 5 min to assess cell viability. Finally, cells were transferred to a Bürker chamber and counted by light microscope. Dead cells were defined as those stained with the dye.

\subsection{Total extracts}

Cells were seeded in $75 \mathrm{~cm}^{2}$ plates and treated as required. Cells were then re-suspended in lysis buffer containing $20 \mathrm{mM}$ Tris- $\mathrm{HCl}(\mathrm{pH} 7.4), 150 \mathrm{mM} \mathrm{NaCl}, 5 \mathrm{mM}$ ethylenediaminetetraacetic acid (EDTA), $0.1 \mathrm{mM}$ phenylmethyl-sulfonyl fluoride (PMSF), $0.05 \%$ aprotinin, $0.1 \%$ Igepal and then incubated for $30 \mathrm{~min}$ at $4{ }^{\circ} \mathrm{C}$. The suspension was centrifuged for $25 \mathrm{~min}$ at $12000 \mathrm{rpm}$ and the supernatant was saved as the total extract. Protein levels were measured using a commercially available assay (Protein Assay Kit 2, Bio-Rad Laboratories, Milano, Italy) with bovine serum albumin as a standard.

\subsection{Western blotting}

Total extracts were then subjected to sodium dodecyl sulphate-polyacrylamide gel electrophoresis on 12,10 or $7.5 \%$ acrylamide gels (Bio-Rad Laboratories, Milano, Italy). The blots were incubated first with desired primary antibody and then with peroxidase-conjugated anti-mouse or anti-rabbit antibodies in Tris-buffered saline-Tween containing 2\% (wt/vol) non-fat dry milk, and developed 
with the enhanced chemiluminescence reagent. Band intensities were quantified by densitometry (VersaDoc Imaging System 3000, Bio-Rad Laboratories, Milano, Italy) and the expression of proteins was reported as a proportion of $\beta$-actin protein expression to monitor gel loading.

\subsection{Morphological analysis}

For indirect immunofluorescence, cells were seeded on 6-well culture plates, allowed to adhere for $24 \mathrm{~h}$ and then treated. After treatment, the cells were fixed and permeabilized with methanol/acetone $(1: 1, \mathrm{v} / \mathrm{v})$. Cells were then incubated with polyclonal antibodies for COX-2, Ecadherin, N-cadherin, or SNAI1, or with monoclonal antibodies for $\beta$-catenin. Immune positivity was revealed by means of appropriate Cy3-conjugated secondary antibody and cells were then stained with Hoechst to detect nuclei. After PBS washings, the slides were mounted with $\mathrm{H}_{2} \mathrm{O} /$ glycerol (1:1) and viewed under a fluorescence microscope (Carl Zeiss, Jena, Germany).

Immunohistochemistry was performed on sections from archived paraffin-included specimens obtained from patients with breast cancer diagnosed during the period 2010-2011 in A.O. Ordine Mauriziano, Pathology Unit, Torino, Italy. Briefly, after microwave antigen retrieval, sections (2 $\mu \mathrm{m}$ thick) were incubated with rabbit polyclonal antibodies against COX-2, E-cadherin, HIF-2 $\alpha$, SNAI1 and HO-1 (final dilutions: 1:50, 1:100, 1:100, 1:50 and 1:400, respectively) and then incubated with EnVision HRP-labelled (DAKO Corporation, Carpinteria, CA, USA) antibodies directed against rabbit antigen [26]. Immunohistochemical reactions were visualized by 3'diaminobenzidine tetrahydrochloride as chromogen substrate. Negative controls were performed by replacing the respective primary antibodies by isotype- and concentration-matched irrelevant antibody.

\subsection{Invasion assay}

Cell invasion was analyzed by employing Boyden chambers equipped with $8 \mu \mathrm{m}$ porosity polyvinylpyrrolidone-free polycarbonate filters that were coated with $50 \mu \mathrm{g} / \mathrm{ml}$ of Matrigel solution. The filters were then fixed in ice-cold methanol and stained with crystal violet solution. Cell invasion was quantified by counting, with a Zeiss microscope (Oberkochen, Germany) equipped 
with bright-field optics (400x final magnification), crystal violet-stained cells that invaded Matrigel. For each filter, cells in 10 randomly chosen fields were counted and expressed as the number of invading cells per high-power field.

\subsection{Zimography}

Analysis of the activity of MMPs was performed on culture medium by means of gelatin zymography in $0.1 \%$ gelatin- $30 \%$ acrylamide gels. Equal amounts of culture medium were loaded in each lane properly diluted in Sample Buffer $2 \mathrm{X}(0.5 \mathrm{M}$ Tris $\mathrm{HCl} \mathrm{pH}$ 6.8, glycerol, $10 \%$ SDS, $0.1 \%$ Bromophenol Blue, distilled water, without $\beta$-mercaptoethanol) and the overall procedure was performed as previously described [21]. Gelatinolytic activity of MMPs was detected as the clear zones of lysis against a blue background. Protein molecular weight marker was also run simultaneously with the test samples. Images were digitalized by mean of VersaDoc 4000 and analyzed with Quantity One software (Bio-Rad Laboratories, Milano, Italy).

\subsection{Detection of intracellular generation of ROS}

Generation of intracellular ROS was analyzed by the semi-quantitative technique based on the use of the $2^{\prime}, 7^{\prime}$-dichlorodihydrofluorescein diacetate (DCFH-DA). MDA-MB-231 cells were seeded in 12-well culture plates $\left(10^{5}\right.$ cells per well). For comparative purposes, cells were treated or not with $50 \mu \mathrm{M}$ hydrogen peroxide $\left(\mathrm{H}_{2} \mathrm{O}_{2}\right.$, positive control) for 15 min under normoxic conditions. ROS (green fluorescence) has been detected by using the conversion of DCFH-DA (used at $5 \mu \mathrm{M}$ concentration for $15 \mathrm{~min}$ ) into the corresponding fluorescent derivative [27]. Cells were observed and photographed under a Zeiss fluorescence microscope (600x final magnification).

\subsection{Statistical analysis}

Statistical significance of differences between independent groups were analyzed using the one-way ANOVA test with Bonferroni post hoc multiple comparisons. All values are expressed as means \pm $\mathrm{SD}$, and differences were considered significant at $\mathrm{p}<0.05$. 


\section{RESULTS}

\subsection{COX-2 expression and prostaglandin production}

MCF-7 human breast tumour cell line, that lacks endogenous COX-2 expression, was transiently transfected with either human COX-2 cDNA or the empty vector (control ${ }_{\mathrm{VECTOR}}$ ) by employing Xtreme GENE HP DNA transfection reagent, chosen for its high efficiency of transfection and for low cytotoxicity (Fig. 1A). After transfection, the cells were allowed to grow for $48 \mathrm{~h}$, then the presence of COX-2 protein was analyzed by western blotting. As shown in Figure 1B, COX-2 protein level was strongly increased in transfected MCF-7 cells, while the COX-2 band was not detectable in control and in controlveCTOR MCF-7 cells. The overexpression of COX-2 was also confirmed by indirect immune fluorescence that revealed the appearance of cytoplasmic COX-2 immune reactivity only in transfected cells (Fig. 1C).

In order to obtain a better estimate of the COX-2 protein level and of its enzyme activity, the production of the $\mathrm{PGE}_{2}$, the major product of $\mathrm{COX}$-2-initiated arachidonic acid metabolism, was measured in the culture medium with an EIA immunoassay. As expected, only COX-2overexpressing MCF-7 cells produced high levels of $\mathrm{PGE}_{2}$ whereas its release was negligible in both control cells (Fig. 1D) and in controlveCTOR cells (data not shown). These results indicate that in transfected cells the overexpressed COX-2 protein is biologically active.

\subsection{EMT features in COX-2- overexpressing cells}

The morphological changes characteristic of cells undergoing EMT are accompanied by a shift in gene expression from an epithelial to a mesenchymal repertoire. To determine whether COX-2 promotes such a shift, both the expression and cellular distribution of selected EMT markers have been investigated by western blotting and immune fluorescence. In COX-2-overexpressing cells, Ecadherin and $\beta$-catenin protein levels are significantly diminished with respect to control and control $_{\text {VECTOR }}$ cells (Fig. 2A). Accordingly, positivity for E-cadherin and $\beta$-catenin under immune fluorescence was similarly reduced in COX-2-overexpressing MCF-7 cells versus parental MCF-7 cells, then essentially confirming immunoblot findings (Fig. 2B). Down-regulation of E-cadherin 
expression in COX-2-overexpressing MCF-7 cells is associated with an increased expression level of the transcriptional repressor SNAI1 (Fig. 2A), that is also significantly localized in the nuclei of transfected cells (Fig. 2B).

Concomitantly, transfected cells presented an increased expression of the mesenchymal markers Ncadherin and vimentin (Fig. 2A) and again N-cadherin increased expression was confirmed by immune fluorescence analysis (Fig. 2B).

\subsection{Roles of MAPK and PI3K/Akt in EMT induction}

The implementation of EMT also depends on the concomitant activity of a variety of signaltransduction pathways, including ERK/MAPK and PI3K/Akt which, in turn, are also relevant for cell proliferation and survival [16]. Therefore, we determined the possible involvement of ERK1/2 and Akt kinase pathways in COX-2-dependent induction of EMT. Although literature data suggest that activation of ERK/MAPK is usually required for a complete EMT in a variety of tumour models [17], in transfected MCF-7 cells no significant increase in the level of the phosphorylated isoforms of ERK was observed (Fig. 3A). Whether PI3K/Akt pathway is concerned, the expression levels of the active form of Akt (p-Akt), the most important downstream effector of PI3K signalling, was significantly higher in transfected MCF-7 cells as compared to control and control $_{\text {VECTOR }}$ cells (Fig. 3A). Since this signalling component has been described, following activation, to phosphorylate and then inhibit GSK3 $\beta$ [28], a critical signalling cross-road in maintaining the epithelial phenotype, we evaluated the level of expression of GSK3 $\beta$ in transfected MCF-7 cells. Western blotting analysis revealed that COX-2 overexpression resulted in an apparent increase of the phosphorylated (inactive) form of GSK3 $\beta$ (Fig. 3A), a scenario which is known to be potentially consistent with the acquisition of mesenchymal morphology.

\subsection{COX-2 modulation of cell invasiveness}

We next evaluated whether COX-2 overexpression might be associated with an increase in cell invasiveness. Interestingly, we found that COX-2-overexpressing MCF-7 cells were three-fold more invasive than parental cells (Fig. 3B), and the increased invasiveness was accompanied by a 
relevant increase of matrix metalloproteinase (MMP)-9 release in the culture medium with respect to parental cells (Fig. 3C), whereas MMP-2 was undetectable (not shown). MMPs are secreted proteolytic enzymes important in promoting invasion through the extracellular matrix. Among them, gelatinases MMP-2 and MMP-9 mediate tumour invasion and metastasis through the degradation of collagen IV and their overexpression has been associated with a more aggressive tumour phenotype.

\subsection{Inhibition of EMT with a COX-2 inhibitor}

Next, we evaluated the role of COX-2 in inducing EMT and then increased invasiveness by an alternative experimental approach involving the use of a COX-2 specific inhibitor, celecoxib. Accordingly, COX-2-overexpressing MCF-7 cells as well as parental MCF cells were treated with celecoxib at 10, 25 and $50 \mu \mathrm{M}$ concentrations and its effect on cell viability was analyzed. Results obtained confirmed our previous findings [4], as in both MCF cells (Fig. 4A) and COX-2overexpressing MCF-7 cells (Fig. 4B) the drug can induce a progressive decrease in cell viability, with the most relevant effects on cell viability being detected in the presence of $50 \mu \mathrm{M}$ celecoxib; as a consequence, $50 \mu \mathrm{M}$ celecoxib was not further used in the other experiments.

When transfected cells were exposed to celecoxib at 10 and $25 \mu \mathrm{M}$ concentrations in order to evaluate effects on selected EMT markers (Fig. 4C), celecoxib was found to result in an apparent increase in E-cadherin and $\beta$-catenin levels as well as in a reduction of $\mathrm{N}$-cadherin levels as compared to transfected only cells. Remarkably, results shown in Figure 4D indicate that celecoxib treatment was also able to reduce cell invasiveness in a dose-dependent fashion, a finding that supports the notion that $\mathrm{COX}-2$ overexpression is required to maintain the increased invasive potential. Moreover, it should be noted that apparent recovery of epithelial phenotype as well as inhibition of invasiveness were already evident in the presence of $10 \mu \mathrm{M}$ celecoxib, a concentration unable to affect viability in either parental or transfected MCF-7 cells.

\subsection{Hypoxia modulation of COX-2 expression and EMT phenotype}


A further goal of this study was to investigate whether exposure to hypoxic conditions may affect COX-2 expression and the related induction of EMT. For this purpose, parental MCF-7, COX-2overexpressing MCF-7 and COX-2-expressing, highly invasive MDA-MB-231 human breast cancer cells were exposed to $3 \%$ oxygen for $2 \mathrm{~h}, 4 \mathrm{~h}, 8 \mathrm{~h}$ and $24 \mathrm{~h}$.

The intracellular levels of HIF-1 $\alpha$, evaluated in order to follow the hypoxic response, were similar in both parental and transfected MCF-7 cells: HIF-1 $\alpha$ levels were found increased already after $2 \mathrm{~h}$, peaked at $4 \mathrm{~h}$ and were still higher than those detected under normoxic condition at $8 \mathrm{~h}$ and $24 \mathrm{~h}$ (Fig. 5A,B).

In parental MCF-7 cells, in which COX-2 is not expressed, changes in HIF-1 $\alpha$ levels were paralleled by increase of $\mathrm{N}$-cadherin levels (Fig.5A) while COX-2 expression was not induced (data not shown). Interestingly, exposure to hypoxic conditions resulted in a further stimulation of COX2 expression and in an induction of EMT-related changes in transfected MCF-7 cells, as suggested by down-regulation of $\beta$-catenin and E-cadherin, and up-regulation of the mesenchymal marker $\mathrm{N}$ cadherin (Fig. 5B). These changes were also paralleled by significant increase of the invasive ability (Fig. 5C) and MMP-9 release (Fig.5D).

The effects induced by hypoxia were then compared with those detected in COX-2-expressing MDA-MB-231 human breast cancer cells. Under normoxic conditions, MDA-MB-231 cells present a fibroblastoid-like appearance and are characterised by lack of E-cadherin expression [29] and, at the same time, by increased levels of N-cadherin, that is an EMT-related overall phenotype/behaviour homologous to the one described in transfected MCF-7 cells. In hypoxic condition, MDA-MB-231 cells expressed HIF-1 $\alpha$ in a time-dependent manner, as it was increased at $2 \mathrm{~h}$, peaked at 4 and $8 \mathrm{~h}$ and decreased after $24 \mathrm{~h}$ (Fig. 6A). Similarly to the scenario detected in transfected MCF-7 cells, HIF-1 $\alpha$ level profile was apparently correlated with up-regulation of both COX-2 and the mesenchymal marker N-cadherin, as well as with down-regulation of the epithelial marker $\beta$-catenin (Fig. 6A). Once again, exposure of mesenchymal-like MDA-MB-231 cells to 
hypoxia resulted in a significant increase of the invasive ability (Fig. 6B) and of MMP-2 and MMP9 release in the extracellular medium (Fig.6C).

\subsection{COX-2 modulation of ROS production and heme oxygenase expression}

We next performed experiments in order to investigate whether COX-2 overexpression may be critical for intracellular generation of reactive oxygen species (ROS) in human breast cancer cells. By employing the DCFH-DA morphological technique, we observed that untreated COX-2expressing MDA-MB-231 cells were already characterized by relevant levels of intracellular ROS (see positive fluorescence of cells in Fig. 7A), that were not modified by addition of hydrogen peroxide in the medium. Of interest, pre-treatment of the same cells with the selective COX-2 inhibitor celecoxib resulted in a significant inhibition of ROS generation (i.e., significant reduction in the number of fluorescent cells). Since these data suggested that COX-2 was significantly contributing to intracellular ROS generation, we next analyzed the potential relationships between COX-2-related ROS production and the expression of heme oxygenase-1 (HO-1), a cytoprotective enzyme with antioxidant activity which is known to be up-regulated in response to increased oxidative stress [30]. In agreement with data obtained with DCFH-DA technique, untreated COX-2expressing MDA-MB-231 cells were characterized by the presence of readily detectable HO-1 protein levels. Moreover, HO-1 protein levels, unmodified following exposure to $\mathrm{H}_{2} \mathrm{O}_{2}$, were drastically reduced in cells pre-treated for $24 \mathrm{~h}$ with the selective COX-2 inhibitor celecoxib (Fig. 7B), thus confirming the involvement of COX-2 in ROS generation.

\subsection{COX-2, E-cadherin, HIF-2 $\alpha$, SNAII and HO-1 tissue expression}

In order to investigate whether data obtained in cell culture may have an in vivo counterpart, we next performed immunohistochemistry for COX-2, E-cadherin, HIF-2 $\alpha$, SNAI1 and HO-1 on serial sections obtained from either three normal breast tissue specimens or twelve specimens obtained from patients with ductal breast cancer (Fig. 8). COX-2 immune positivity, which was detectable at low level in normal breast tissue, was found to have a predominant cytoplasmic and perinuclear localization in breast cancer epithelial cells, according to previous data from our laboratory [5] and 
other research groups [31]. By comparing immune histochemical images obtained in normal breast tissue and ductal breast cancer, malignant breast cells differed from normal breast epithelial cells not only for COX-2-positivity but also for a number of additional significant features, including: a) strongly increased positive nuclear staining for the EMT-related transcription factor SNAI1 and the hypoxia-related transcription factor HIF-2 $\alpha$; b) significantly decreased positive staining, diffuse in the cytoplasm, for E-cadherin, as compared to the very strong positive staining observed in normal breast specimens; c) strong positive cytoplasmic staining for HO-1 (absent in epithelial cells of normal breast). These morphological observations are consistent with the concept that COX-2positive malignant cells in vivo may not only express EMT-related features but may also face conditions of tissue hypoxia and intracellular ROS generation.

\section{DISCUSSION}

The aim of the present study was to investigate whether changes in COX-2 expression may affect epithelial to mesenchymal transition (EMT) and invasive potential of human breast cancer cells. As a first approach, we successfully transfected slow growing, non-invasive COX-2 null MCF-7 human breast tumour cells in order to obtain cancer cells that transiently overexpressed COX-2 and to analyse then COX-2 putative role in the modulation of phenotypic features and invasiveness.

One of the most interesting changes we detected in transfected MCF-7 cells was the apparent loss of selected epithelial features and the acquisition of mesenchymal-like phenotypic changes that are widely considered as hallmarks of EMT. At first, transfected MCF-7 cells exhibited an increase of $\mathrm{N}$-cadherin and vimentin expression accompanied by a reduction of E-cadherin and $\beta$-catenin levels. E-cadherin is a key member of a family of functionally related transmembrane glycoproteins that mediate intercellular adhesion. The cytoplasmic terminal tail of E-cadherin links specifically to $\beta$-catenin, that, in turn, binds directly to cytoskeletal actin leading to the assembly of cell-cell junctions. A loss of E-cadherin expression or function was frequently shown in many different types of tumours, where it accompanies the invasiveness and metastatic behaviour of malignant 
cells $[14-16,22,32]$. It is well known that down-regulation and relocation of E-cadherin/ $\beta$-catenin complex is critical in the EMT process, leading to $\beta$-catenin release from the cell membrane followed by accumulation in the cytoplasm and translocation to the nucleus where it promotes cell proliferation. These data are consistent with and further expand preliminary data published earlier that were suggesting changes into a fibroblastoid morphology and increased vimentin expression in MCF-10 cells stably transfected to overexpress COX-2 [33].

According to literature data [18], down-regulation of E-cadherin in transfected MCF-7 cells is related to the overexpression of SNAI1, a zinc finger transcription factor able to directly repress Ecadherin expression following its binding to the E-boxes of the human E-cadherin promoter [16,19]. Indeed, overexpression of SNAI1 predicts for a more aggressive tumour phenotype [34] and studies on breast cancer indicated that SNAI1 overexpression is associated with increased lymph node involvement, invasiveness and metastatic potential [35] as well as with decreased recurrence-free survival [36] through mechanisms that remains largely unresolved.

Moreover, SNAI1 up-regulation detected in transfected MCF-7 cells is likely to be related to the increase of the phosphorylated form (i.e., inactive form) of GSK3 $\beta$. Accumulating literature evidences indicate that GSK3 $\beta$ activity is critical in maintaining the epithelial phenotype, with GSK3 $\beta$-dependent SNAI1 binding and phosphorylation facilitating its proteasomal degradation $[32,37,38]$. Accordingly, inhibition of GSK3 $\beta$ upon phosphorylation can promote the stabilization and the nuclear translocation of SNAI1, and the subsequent induction of EMT [16,32,39].

Concerning mechanisms able to relate COX-2 overexpression and EMT, recent reports [40] indicate that $\mathrm{COX}-2 / \mathrm{PGE}_{2}$ pathway may be responsible for activation of both PI3K/Akt and ERK1/2 pathways via the prostaglandin $\mathrm{EP}_{4}$ receptor, thus resulting in increased growth, motility, and resistance to apoptosis of cancer cells. Data in this study, however, indicate that in COX-2overexpressing MCF-7 cells only the levels of phospho-Akt were up-regulated whereas no apparent increase of phosphorylated (i.e., active) ERK1/2 isoforms was detected. This is consistent with literature data $[41,42]$ indicating that PI3K/Akt signalling plays a prominent role in inducing and 
maintaining EMT and also suggest that in transfected MCF-7 cells overexpression of COX-2, through activation of PI3K/Akt, can result in the reduction of the active form of GSK3ß and modulation of EMT-related signals, including up-regulation of SNAI1 and consequent repression of E-cadherin gene transcription and EMT induction. This interpretation is sustained by the following major findings and considerations: a) transfected MCF-7 cells exhibited a more invasive phenotype with respect to parental cells and possessed extracellular matrix degrading activity that was likely due to the release of the matrix metalloproteinase 9; b) the overall phenotype of transfected MCF-7 cells was consistent with the one exhibited by the more aggressive and invasive MDA-B231 cells, which are known to normally overexpress COX-2; c) EMT related changes and increased invasiveness were at least partly prevented by pre-treatment of COX-2 expressing human breast cells with celecoxib, a well known selective COX-2 inhibitor.

Whether the critical role of COX-2 in EMT induction from a mechanistic point of view is concerned, current literature offers a number of inter-related and established concepts. First, COX2 has been suggested to be up-regulated in colorectal and lung cancer cells by hypoxic conditions through HIF-1-dependent mechanisms [14,23,24], and that such an induction can enhance cell motility and invasiveness. This is potentially relevant since the presence of hypoxic areas within the neoplastic mass represents a major and common feature of clinically relevant solid malignant tumours [43]. Moreover, hypoxia is currently believed to provide a strong selective pressure able to eventually favour proliferation and survival of the most aggressive malignant cells and the detection of hypoxic areas within a neoplastic mass is considered an independent prognostic indicator of poor outcome with a significant risk to develop metastasis that may escape therapy $[12,44]$. Relevant to this study, hypoxia has also been shown to trigger epithelial-to-mesenchymal transition (EMT) in human malignant cancer cells of different origin [21]. Along these lines, in the present study we were able to confirm that exposure of COX-2-overexpressing MCF-7 and COX-2 expressing, highly invasive MDA-MB-231 human breast cancer cells to hypoxia resulted in a further (i.e., two fold) increase in the expression of COX-2 protein as well as in an exacerbation of typical EMT- 
related changes (down regulation of $\beta$-catenin, up-regulation of $\mathrm{N}$-cadherin) coupled to increased release of MMPs and overall invasive ability. These results then corroborate the relevant additional concept that hypoxic conditions can effectively enhance COX-2 expression levels and through this event further promote EMT induction and invasiveness. Such a scenario is compatible with results obtained by immunohistochemistry performed on thin serial sections obtained from normal breast tissue and breast cancer specimens (Fig. 8). This morphological analysis showed that malignant epithelial cells of ductal breast cancer were characterized by positive immune-staining for COX-2 as well as by changes consistent with ongoing EMT (decreased staining for E-cadherin and increased nuclear staining for SNAI1). Moreover, epithelial breast cancer cells but not epithelial cells in normal breast, showed cytoplasmic immune positivity for $\mathrm{HO}-1$ as well as a very significant increase in nuclear positivity for HIF-2 $\alpha$. Taken together, these data suggest that in vivo COX-2-positive cells were likely not only to express EMT-related features but also to face conditions of tissue hypoxia and intracellular reactive oxygen species (ROS) generation. These data are also consistent with previous results suggesting the existence of a statistically significant association between COX-2 and human breast cancer-related clinic-pathological parameters associated with poor prognosis, such as size, grading, extent of primary tumor, nodal status and vascular invasion [5].

A final relevant concept should be recalled that may further interconnect COX-2 and hypoxia. COX-2 up-regulation during hypoxia has been associated with an increase in $\mathrm{PGE}_{2}$ levels [24] and the latter has been proposed to play a role in the promotion of cell survival under hypoxic conditions, critical for tumour cells to propagate and progress [12]. Recent studies reported that activation of COX-2 led to increased intracellular generation of ROS mechanistically linked to EMT induction in prostate cancer cells, an event efficiently prevented by either antioxidant treatment or by COX-2 silencing $[45,46,47]$.

The possible critical role of ROS generated by COX-2 has been revealed in the present study concerning human breast cancer cells. Indeed, COX-2 expressing MDA-MB-231 cells produced 
abundant levels of ROS, which were significantly reduced by the selective COX-2 inhibitor celecoxib. Accordingly, celecoxib pre-treatment was also able to down-regulate the expression of the redox-sensitive enzyme HO-1, which is overexpressed in untreated MDA-MB-231 cells (this study) as well as in other cancer cells [30]. In keeping with a critical role of ROS released by COX2, our results suggest that COX-2 up-regulation can contribute to ROS generation in breast cancer cells. Furthermore, since celecoxib was reported in our study to prevent EMT changes and invasiveness related to $\mathrm{COX}-2$ expression, it is conceivable that COX-2-related induction of EMT and enhancement of cancer cell invasiveness may significantly depend also on COX-2-dependent intracellular production of ROS.

According to this hypothesis, a recent report highlighted that cancer-associated macrophages (CAM), through a IL-1 $\beta$-dependent and ROS-mediated induction of COX-2 expression in HCC1954 breast cancer cells, resulted in an enhancement in their aggressiveness [48]. Although EMT was not specifically investigated, the data from the latter study suggest than that also stimuli released from cancer associated macrophages may contribute to COX-2 expression again in a redox-related way.

\subsection{Conclusions}

COX-2 up-regulation has been reported in a number of different tumour types [49], and there has been a great deal of interest in attempting to achieve additional understanding of the regulatory networks that control COX-2-related effects. In this scenario, the results obtained in our study allowed to gain additional insights into the biological roles of COX-2 in human breast cancer cell behaviour suggesting a direct link between COX-2 overexpression and invasive ability. COX-2related induction of EMT and increased invasiveness in breast cancer cells, as proposed from a recent study in prostate cancer cells, is likely to be also significantly related to COX-2-dependent intracellular generation of ROS, a scenario which is likely to be emphasized by either hypoxic conditions or other signals (growth factors, cyto- and chemokines) from cancer microenvironment (Fig. 9). 
Future studies into the emerging players within the $\mathrm{COX}-2 / \mathrm{PGE}_{2}$ pathway may reveal novel approaches for more safely targeting this pathway for both cancer chemoprevention and therapy.

\section{DISCLOSURE OF POTENTIAL CONFLICTS OF INTEREST}

Authors involved in the present study have no potential conflicts of interest to disclose and they

have received no payment in preparation of this manuscript. The financial support was received from University of Torino, Torino, Italy. 


\section{REFERENCES}

1. Jemal A, Siegel R, Ward E, et al. Cancer statistics. CA Cancer J Clin 2008;58:71-96.

2. Wiwanitkit V. Combination of EGFR and COX-2 inhibitors in breast cancer patient. Tumour Biol 2012;33:1261.

3. Park BW, Park S, Park HS, et al. Cyclooxygenase-2 expression in proliferative Ki-67positive breast cancers is associated with poor outcomes. Breast Cancer Res Treat 2012;133:741-751.

4. Bocca C, Bozzo F, Bassignana A, et al. Antiproliferative effects of COX-2 inhibitor celecoxib on human breast cancer cell lines. Mol Cell Biochem 2011;350:59-70.

5. Miglietta A, Toselli M, Ravarino N, et al. COX-2 expression in human breast carcinomas: correlation with clinicopathological features and prognostic molecular markers. Expert Opin Ther Targets 2010;14:655-664.

6. Singh B, Lucci A. Role of cyclooxygenase-2 in breast cancer. J Surg Res 2002;108:173-179.

7. Harris RE. Cyclooxygenase-2 (cox-2) blockade in the chemoprevention of cancers of the colon, breast, prostate, and lung. Inflammopharmacology 2009;17:55-67.

8. Imada T, Matsuoka J, Nobuhisa T, et al. COX-2 induction by heparanase in the progression of breast cancer. Int J Mol Med 2006;17:221-228.

9. Singh-Ranger G, Salhab M, Mokbel K. The role of cyclooxygenase-2 in breast cancer: review. Breast Cancer Res Treat 2008;109:189-198.*Relevant review outlining COX-2 expression as a fundamental step in breast cancer pathogenesis

10. Liotta LA, Kohn EC. The microenvironment of the tumour-host interface. Nature 2001;411:375-379.

11. Semenza GL. Targeting HIF-1 for cancer therapy. Nat Rev Cancer 2003;3:721-732.

12. Vaupel P, Mayer A. Hypoxia in cancer: significance and impact on clinical outcome. Cancer Metastasis Rev 2007;26:225-239. 
13. Höckel M, Vaupel P. Tumor hypoxia: definitions and current clinical, biologic, and molecular aspects. J Natl Cancer Inst 2001;93:266-276. *Relevant review summarizing the fundamental role for hypoxia in the progression of solid tumors

14. Bocca C, Bozzo F, Cannito S, et al. Celecoxib inactivates epithelial-mesenchymal transition stimulated by hypoxia and/or epidermal growth factor in colon cancer cells. Mol Carcinog 2012;51:783-795.

15. Jiang J, Tang YL, Liang XH. EMT: a new vision of hypoxia promoting cancer progression. Cancer Biol Ther 2011;11:714-723.

16. Tiwari N, Gheldof A, Tatari M, et al. EMT as the ultimate survival mechanism of cancer cells. Semin Cancer Biol 2012;22:194-207.

17. Tse JC, Kalluri R. Mechanisms of metastasis: epithelial-to-mesenchymal transition and contribution of tumor microenvironment. J Cell Biochem 2007;101:816-829.

18. Foroni C, Broggini M, Generali D, et al. Epithelial-mesenchymal transition and breast cancer: role, molecular mechanisms and clinical impact. Cancer Treat Res 2012;38:689-697. ** Relevant study focusing on the physiological and pathological role of EMT process, its molecular related network, its putative role in the metastatic process and its implications in response/resistance to the current and/or new approaching drugs in the clinical management of breast cancer.

19. Thiery JP, Acloque H, Huang RY, et al. Epithelial-mesenchymal transitions in development and disease. Cell 2009;139:871-890.

20. Lundgren K, Nordenskjöld B, Landberg G. Hypoxia, Snail and incomplete epithelialmesenchymal transition in breast cancer. Br J Cancer 2009;101:1769-1781.

21. Cannito S, Novo E, Compagnone A, et al. Redox mechanisms switch on hypoxia-dependent epithelial-mesenchymal transition in cancer cells. Carcinogenesis 2008;29:2267-2278.

22. Cannito S, Novo E, di Bonzo LV, et al. Epithelial-Mesenchymal Transition: From Molecular Mechanisms, Redox Regulation to Implications in Human Health and Disease. 
Antioxid Redox Signal 2010;12:1383-1430. *Relevant review summarising the major relevant features of EMT and the signaling mechanisms that are known to regulate or affect the process, including the role for oxidative stress and reactive oxygen species (ROS)

23. Csiki I, Yanagisawa K, Haruki N, et al. Thioredoxin-1 modulates transcription of cyclooxygenase-2 via hypoxia-inducible factor-1alpha in non-small cell lung cancer. Cancer Res 2006;66:143-150.

24. Kaidi A, Qualtrough D, Williams AC, et al. Direct transcriptional up-regulation of cyclooxygenase-2 by hypoxia-inducible factor (HIF)-1 promotes colorectal tumor cell survival and enhances HIF-1 transcriptional activity during hypoxia. Cancer Res 2006;66:6683-6691.*Relevant study outlining COX-2 as a direct target for HIF-1 in colorectal tumor cells, with implication for cancer cell survival and angiogenesis

25. Liu XH, Rose DP. Differential expression and regulation of cyclooxygenase- 1 and-2 in two human breast cancer cell lines. Cancer Res 1996;56:5125-5127.

26. Novo E, Povero D, Busletta C, et al. The biphasic nature of hypoxia-induced directional migration of activated human hepatic stellate cells. J Pathol 2012;226:588-597.

27. Rezvani HR, Dedieu S, North S, et al. Hypoxia inducible factor $1 \alpha$, a key factor in the keratinocyte response to UVB exposure. J Biol Chem 2007;282:16413-16422.

28. Jope RS, Johnson GV. The glamour and gloom of glycogen synthase kinase-3. Trends Biochem Sci 2004;29:95-102.

29. Chao YL, Shepard CR, Wells A. Breast carcinoma cells re-express E-cadherin during mesenchymal to epithelial reverting transition. Mol Cancer 2010;9:179-197.

30. Grochot-Przeczek A, Dulak J, Jozkowicz A. Haem oxygenase-1: non-canonical roles in physiology and pathology. Clin Sci (Lond) 2012;122:93-103. 
31. Maroni P, Matteucci E, Luzzati A, et al. Nuclear co-localization and functional interaction of COX-2 and HIF-1 $\alpha$ characterize bone metastasis of human breast carcinoma. Breast Cancer Res Treat 2011;129:433-450.

32. Bastid J. EMT in carcinoma progression and dissemination: facts, unanswered questions, and clinical considerations. Cancer Metastasis Rev 2012;31:277-283.

33. $\mathrm{Lu} \mathrm{S}$, Yu G, Zhu Y, et al. Cyclooxygenase-2 overexpression in MCF-10F human breast epithelial cells inhibits proliferation, apoptosis and differentiation, and causes partial transformation. In J Cancer 2005;116:847-852.

34. Francí C, Gallén M, Alameda F, et al. Snail1 protein in the stroma as a new putative prognosis marker for colon tumours. PLoS One 2009;4:e5595.

35. Blanco MJ, Moreno-Bueno G, Sarrio D, et al. Correlation of Snail expression with histological grade and lymph node status in breast carcinomas. Oncogene 2002;21:32413246.

36. Moody SE, Perez D, Pan TC, et al. The transcriptional repressor snail promotes mammary tumor recurrence. Cancer Cell 2005;8:197-209.*Relevant clinical study that outlines that Snail expression levels are sufficient to promote mammary tumor recurrence in vivo, and that high levels of Snail predict decreased relapse-free survival in women with breast cancer

37. Doble BW, Woodgett JR. Role of glycogen synthase kinase-3 in cell fate and epithelialmesenchymal transitions. Cells Tissues Organs 2007;185:73-84.

38. Acloque H, Adams MS, Fishwick K, et al. Epithelial-mesenchymal transitions: the importance of changing cell state in development and disease. J Clin Invest 2009;119:14381449.

39. Zhou BP, Deng J, Xia W, et al. Dual regulation of Snail by GSK-3beta-mediated phosphorylation in control of epithelial-mesenchymal transition. Nat Cell Biol 2004;6:931940. 
40. Greenhough $\mathrm{A}$, Smartt $\mathrm{HJ}$, Moore $\mathrm{AE}$, et al. The COX-2/PGE 2 pathway: key roles in the hallmarks of cancer and adaptation to the tumour microenvironment. Carcinogenesis 2009;30:377-386. *Relevant study focusing on the emerging regulators of COXprostaglandin signaling and novel roles for $\mathrm{COX}-2 / \mathrm{PGE}_{2}$ signaling in the tumour microenvironment.

41. Smirnova T, Zhou ZN, Flinn RJ, et al. Phosphoinositide 3-kinase signaling is critical for ErbB3-driven breast cancer cell motility and metastasis. Oncogene 2012;31:706-715.

42. Liu XH, Kirschenbaum A, Lu M, et al. Prostaglandin $\mathrm{E}_{2}$ Induces Hypoxia-inducible Factor$1 \alpha$ Stabilization and Nuclear Localization in a Human Prostate Cancer Cell Line. J Biol Chem 2002;277:50081-50086.

43. Brahimi-Horn MC, Chiche J, Pouysségur J. Hypoxia and cancer. J Mol Med 2007;85:13011307.

44. Rankin EB, Giaccia AJ. The role of hypoxia-inducible factors in tumorigenesis. Cell Death \& Differ 2008;15:678-685.

45. Giannoni E, Parri M, Chiarugi P. EMT and oxidative stress: a bidirectional interplay affecting tumor malignancy. Antioxid Redox Signal 2012;16:1248-1263. *Relevant review outlining the concept that intracellular ROS may regulate EMT through a mechanism involving HIF-1 and COX-2.

46. Giannoni E, Bianchini F, Masieri L, et al. Reciprocal activation of prostate cancer cells and cancer-associated fibroblasts stimulates epithelial-mesenchymal transition and cancer stemness. Cancer Res 2010;70:6945-6956.

47. Giannoni E, Bianchini F, Calorini L, et al. Cancer associated fibroblasts exploit reactive oxygen species through a proinflammatory signature leading to epithelial mesenchymal transition and stemness. Antioxid Redox Signal 2011;14:2361-2371.

48. Hou Z, Falcone DJ, Subbaramaiah K, et al. Macrophages induce COX-2 expression in breast cancer cells: Role of IL-1beta autoamplification. Carcinogenesis 2011;32:695-702. 
49. Subbaramaiah K, Dannenberg AJ. Cyclooxygenase 2: a molecular target for cancer prevention and treatment. Trends Pharmacol Sci 2003;24:96-102. 


\section{TITLES AND LEGENDS TO FIGURES}

FIGURE 1: COX-2 expression, subcellular localization and activity.

MCF-7 cells were transiently transfected by employing X-treme GENE HP DNA transfection reagent.

A: Cell viability was assessed by the trypan blue exclusion test after $48 \mathrm{~h}$ from the transfection. Results are expressed as viable cell number $/ \mathrm{ml}$ and represent mean \pm S.D. of three independent experiments, each performed in triplicate.

B: COX-2 protein expression levels were analyzed by western blotting. Total cell lysates were incubated with polyclonal antibody for COX-2 and then with HRP-conjugated secondary antibody. The blot shown is representative of three independent experiments.

C: COX-2 immune reactivity was detected in MCF-7 cells exposed to anti-COX-2 polyclonal primary antibody followed by anti-mouse Cy3-conjugated secondary antibody. To detect nuclei the cells were stained with Hoechst. The images show the overlaid pictures (400× final magnification) and are representative of three independent experiments.

D: COX-2 activity was detected by measuring the amounts of $\mathrm{PGE}_{2}$ in cell culture medium as described in Materials and Methods. Data are expressed as $\mathrm{pg} / \mathrm{ml} \mathrm{PGE} 2$ and represent mean \pm S.D. of three independent experiments, each performed in triplicate.

FIGURE 2: Expression and subcellular localization of EMT markers in MCF-7 cells under normoxic condition.

A: Total cell lysates were analyzed by western blotting to determine the expression levels of Ecadherin, $\beta$-catenin, N-cadherin, vimentin and SNAI1. The optical density of the bands was determined by densitometry, normalized with respect to that of the corresponding $\beta$-actin band and expressed as arbitrary units relative to the control (S.D. < 10\%). The data are representative of three independent experiments. 
B: The cells were fixed and exposed to anti-E-cadherin, $\beta$-catenin, N-cadherin and SNAI1 primary antibody followed by proper Cy3-conjugated secondary antibody. To detect nuclei the cells were stained with Hoechst. The images show the overlaid pictures (400× final magnification) and are representative of three independent experiments.

FIGURE 3: Analysis of COX-2 related signaling pathway and invasive ability.

A: Total cell lysates were analyzed by western blotting to determine the expression levels of p-Akt p-ERK1/2 and p-GSK3 $\beta$. The optical density of the bands was determined by densitometry, normalized with respect to that of the corresponding Akt, ERK1/2 or $\beta$-actin band and expressed as arbitrary units relative to the control (S.D. $<10 \%)$. The data are representative of three independent experiments.

B: Matrigel invasion was evaluated by employing Boyden chamber's assay; each filter was examined with a Zeiss microscope (400x final magnification) and the number of cells able to invade the matrigel was counted. Data are expressed as the number of cells per high-magnification field and represent the mean of three independent experiments, each performed in triplicate (bars, S.D.). $*_{p}<0.01$ versus values in MCF-7 parental cells (control).

C: MMP-9 release was determined by gelatin zymography of cell-free supernatants obtained from MCF-7 cells maintained for $24 \mathrm{~h}$ under normoxic condition. The zymogram shown is representative of three independent experiments. Gelatinase activity was estimated by densitometry and expressed as arbitrary units relative to normoxic control (S.D. < 10\%).

FIGURE 4: Effect of celecoxib on cell viability, EMT induction and invasive ability.

Control and transfected MCF-7 cells were incubated for $24 \mathrm{~h}$ in the absence or in the presence of celecoxib at the indicated concentrations. 
A,B: Cell viability was assessed by the trypan blue exclusion test in control (A) and transfected (B) MCF-7 cells. Results are expressed as number of viable cells $/ \mathrm{ml}$ and values represent mean \pm S.D. of three independent experiments, each performed in triplicate.

$* p<0.05, * * p<0.001$ versus values in respective control.

C: Total cell lysates were analyzed by western blotting to determine levels of COX-2, E-cadherin, B-catenin and $\mathrm{N}$-cadherin. The optical density of the bands was determined by densitometry, normalized with respect to that of the corresponding $\beta$-actin band and expressed as arbitrary units relative to the control (S.D. $<10 \%)$. The data are representative of three independent experiments.

D: Matrigel invasion was evaluated by employing Boyden chamber's assay; each filter was examined with a Zeiss microscope (400x final magnification) and the number of cells able to invade the matrigel was counted. Data are expressed as the number of cells per high-magnification field and represent the mean of three independent experiments, each performed in triplicate (bars, S.D.).

${ }^{*} p<0.001$ versus values in control cells; ${ }^{\S} p<0.05,{ }^{\S \S} p<0.01$ versus values in transfected cells

FIGURE 5: Effect of hypoxia on EMT induction and invasive ability in control and transfected MCF-7 cells.

A, B: Control (A) and transfected (B) MCF-7 cells were incubated for $0 \mathrm{~h}$ (control), $2 \mathrm{~h}, 4 \mathrm{~h}, 8 \mathrm{~h}$ and $24 \mathrm{~h}$ under hypoxic conditions. Total cell lysates were analyzed by western blotting to determine levels of HIF-1 $\alpha$, $\beta$-catenin, E-cadherin, N-cadherin and COX-2. The optical density of the bands was determined by densitometry, normalized with respect to that of the corresponding $\beta$ actin band and expressed as arbitrary units relative to the control (S.D. $<10 \%$ ). The data are representative of three independent experiments.

C: Control and transfected MCF-7 cells were incubated for $24 \mathrm{~h}$ under hypoxic conditions. Matrigel invasion was evaluated by employing Boyden chamber's assay; each filter was examined with a Zeiss microscope (400x final magnification) and the number of cells able to invade the matrigel 
was counted. Data are expressed as the number of cells per high-magnification field and represent the mean of three independent experiments, each performed in triplicate (bars, S.D.). ${ }^{*} p<0.01$ versus values in normoxic control cells.

D: Control and transfected MCF-7 cells were incubated for $24 \mathrm{~h}$ under normoxic and hypoxic conditions. MMP-9 release was determined by gelatin zymography of cell-free supernatants. The zymograms shown are representative of three independent experiments. Gelatinase activity was estimated by densitometry and expressed as arbitrary units relative to normoxic control (S.D. < $10 \%)$.

FIGURE 6: Effect of hypoxia on EMT induction and invasive ability in MDA-MB-231 cells.

A: MDA-MB-231 cells were incubated for $0 \mathrm{~h}$ (control), $2 \mathrm{~h}, 4 \mathrm{~h}, 8 \mathrm{~h}$ and $24 \mathrm{~h}$ under hypoxic conditions. Total cell lysates were analyzed by western blotting to determine levels of HIF-1 $\alpha$, COX-2, $\beta$-catenin and N-cadherin. The optical density of the bands was determined by densitometry, normalized with respect to that of the corresponding $\beta$-actin band and expressed as arbitrary units relative to the control (S.D. $<10 \%$ ). The data are representative of three independent experiments.

B: The cells were incubated for $24 \mathrm{~h}$ under normoxic and hypoxic conditions. Matrigel invasion was evaluated by employing Boyden chamber's assay; each filter was examined with a Zeiss microscope (400x final magnification) and the number of cells able to invade the matrigel was counted. Data are expressed as the number of cells per high-magnification field and represent the mean of three independent experiments, each performed in triplicate (bars, S.D.).

$*_{p}<0.01$ versus values in normoxic control cells.

C: The cells were incubated for $24 \mathrm{~h}$ under normoxic and hypoxic conditions. MMP-2 and -9 release was determined by gelatin zymography of cell-free supernatants. The zymograms shown are representative of three independent experiments. Gelatinase activity was estimated by densitometry and expressed as arbitrary units relative to normoxic control (S.D. $<10 \%$ ). 
FIGURE 7. Intracellular generation of ROS and heme oxygenase-1 expression in MDA-MB-231 cells.

A: Generation of intracellular ROS was detected by the semi-quantitative technique based on the use of 2',7'-dichlorodihydrofluorescein diacetate (DCFH-DA) in MDA-MB-231 control cells, in MDA-MB-231 cells treated for 15 min with $50 \mu \mathrm{M} \mathrm{H}_{2} \mathrm{O}_{2}$ (positive control) and in MDA-MB-231 cells exposed for $4 \mathrm{~h}$ to celecoxib at 10 and $25 \mu \mathrm{M}$ concentrations. For any condition, the image is obtained by digital overlay of phase contrast image with DCFH-DA positive image. Data in bar graph are expressed as number of fluorescent cells for field and represent the mean \pm S.D. of three independent experiments. ${ }^{*} p<0.01$ versus values in control cells.

B: Heme oxygenase-1 expression was analyzed by western blotting. MDA-MB-231 cells were treated or not for 30 min with $50 \mu \mathrm{M} \mathrm{H}_{2} \mathrm{O}_{2}$ (positive control) or with celecoxib for $24 \mathrm{~h}$ at 10 and 25 $\mu \mathrm{M}$ concentrations. Total cell lysates were incubated with polyclonal antibody for HO-1 followed by HRP-conjugated secondary antibody. The optical density of the bands was determined by densitometry, normalized with respect to that of the corresponding $\beta$-actin band and expressed as arbitrary units relative to the control (S.D. $<10 \%$ ). The data are representative of three independent experiments.

FIGURE 8. Expression of COX-2, EMT markers and heme oxygenase-1 in normal breast tissue and ductal breast cancer (G2, score 7, Elston-Ellis).

Immunohistochemical analysis was performed on serial sections from paraffin-included normal $(n=3)$ breast tissues and breast cancer $(n=12)$ specimens using rabbit polyclonal antibodies against COX-2, E-cadherin, HIF-2 $\alpha$, SNAI1 and HO-1. Immunohistochemical reactions were visualized by 3'-diaminobenzidine tetrahydrochloride as chromogen substrate. Nuclei were counterstained with hematoxylin. Original magnification as indicated. 
FIGURE 9. Schematic representation of the COX-2-related mechanisms potentially leading to mesenchymal morphology in breast cancer cells. COX-2 overexpression, further enhanced by hypoxia, can induce generation of ROS leading to activation of the PI3K/Akt signaling pathway. Activated Akt may cause the inactivation of GSK3 $\beta$ by phosphorylation, which, in turn, can favor translocation of SNAI1 to the nucleus where it functions as a repressor of E-cadherin and then initiates EMT. Celecoxib, as a specific COX-2 inhibitor, can negatively affect EMT induction. 